\title{
Building a Framework for Predictive Science
}

\author{
Michael M. McKerns ${ }^{\ddagger}$, Leif Strand ${ }^{\ddagger}$, Tim Sullivan ${ }^{\ddagger}$, Alta Fang ${ }^{\ddagger}$, Michael A.G. Aivazis
}

\begin{abstract}
Key questions that scientists and engineers typically want to address can be formulated in terms of predictive science. Questions such as: "How well does my computational model represent reality?", "What are the most important parameters in the problem?", and "What is the best next experiment to perform?" are fundamental in solving scientific problems. mystic is a framework for massively-parallel optimization and rigorous sensitivity analysis that enables these motivating questions to be addressed quantitatively as global optimization problems. Often realistic physics, engineering, and materials models may have hundreds of input parameters, hundreds of constraints, and may require execution times of seconds or longer. In more extreme cases, realistic models may be multi-scale, and require the use of high-performance computing clusters for their evaluation. Predictive calculations, formulated as a global optimization over a potential surface in design parameter space, may require an already prohibitively large simulation to be performed hundreds, if not thousands, of times. The need to prepare, schedule, and monitor thousands of model evaluations, and dynamically explore and analyze results, is a challenging problem that requires a software infrastructure capable of distributing and managing computations on large-scale heterogeneous resources. In this paper, we present the design behind an optimization framework, and also a framework for heterogeneous computing, that when utilized together, can make computationally intractable sensitivity and optimization problems much more tractable. The optimization framework provides global search algorithms that have been extended to parallel, where evaluations of the model can be distributed to appropriate large-scale resources, while the optimizer centrally manages their interactions and navigates the objective function. New methods have been developed for imposing and solving constraints that aid in reducing the size and complexity of the optimization problem. Additionally, new algorithms have been developed that launch multiple optimizers in parallel, thus allowing highly efficient local search algorithms to provide fast global optimization. In this way, parallelism in optimization also can allow us to not only find global minima, but to simultaneously find all local minima and transition points -- thus providing a much more efficient means of mapping out a potential energy surface.
\end{abstract}

Index Terms - predictive science, optimization, uncertainty quantification, verification, validation, sensitivity analysis, parallel computing, distributed computing, heterogeneous computing

\section{Introduction}

Recently, a unified mathematical framework for the rigorous construction and solution of uncertainty quantification (UQ) problems was formulated [OSS11]. This framework, called Optimal Uncertainty Quantification (OUQ), is based on the observation that, given a set of assumptions and information about the problem, there exist optimal bounds on the uncertainties. These bounds are

* Corresponding author: mmckerns@caltech.edu

\$ California Institute of Technology

Copyright $@ 2011$ Michael M. McKerns et al. This is an open-access article distributed under the terms of the Creative Commons Attribution License, which permits unrestricted use, distribution, and reproduction in any medium, provided the original author and source are credited. obtained as extreme values of well-defined optimization problems that correspond to extremizing probabilities of failure subject to the constraints imposed by scenarios compatible with the information set.

An accompanying software framework that implements these rigorous UQ/OUQ methods is now posed.

A rigorous quantification of uncertainty can easily require several thousands of model evaluations $f(x)$. For all but the smallest of models, this requires significant clock time -- a model requiring 1 minute of clock time evaluated 10,000 times in a global optimization will take 10,000 minutes $(\sim 7$ days $)$ with a standard optimizer. Furthermore, realistic models are often highdimensional, highly-constrained, and may require several hours to days even when run on a parallel computer cluster. For studies of this size or larger to be feasible, a fundamental shift in how we build optimization algorithms is required. The need to provide support for parallel and distributed computing at the lowest level -- within the optimization algorithm -- is clear. Standard optimization algorithms must be extended to parallel. The need for new massively-parallel optimization algorithms is also clear. If these parallel optimizers are not also seamlessly extensible to distributed and heterogeneous computing, then the scope of problems that can be addressed will be severely limited.

While several robust optimization packages exist [JOP01], [KROOO], there are very few that provide massively-parallel optimization [BMM10], [EKL02], [MAT09] -- the most notable effort being DAKOTA [DAKOT], which also includes methods for uncertainty quantification [DAKUQ]. A rethinking of optimization algorithms, from the ground up, is required to dramatically lower the barrier to massively-parallel optimization and rigorous uncertainty quantification. The construction and tight integration of a framework for heterogeneous parallel computing is required to support such optimizations on realistic models. The goal should be to enable widespread availablility of these tools to scientists and engineers in all fields.

Several of the component pieces of such a framework for predictive science already exist, while a few key pieces must be constructed -- furthermore, these packages must then be assembled and integrated. Python [GVRPY] is a natural integration environment, and is one that readily supports the dynamic nature of working across heterogeneous resources. By requiring this framework be pure-Python, many of the barriers to running on a new platform are removed. multiprocessing [MPROC], mpi 4py [MPI4P], and pp [VVPPP] are selected for communication mechanisms, both due to their high level of feature coverage and their relative ease of installation. NumPy [NUMPY] is used for algorithmic efficiency, and SymPy [SYM11] is used to provide an alternate interface for building constraints. Many of 
the optimization algorithms leverage SciPy [JOP01]; however like the use of Matplotlib [MATPL] for plotting, SciPy is an optional dependency.

This paper will discuss the modifications to the mystic [MHA09] optimization framework required to provide a simple interface to massively parallel optimization, and also to the pathos [MBA10] framework for staging and launching optimizations on heterogeneous resources. These efforts leverage pyre [MAGA1] -- an component integration framework for parallel computing, which has recently been extended to distributed communication and management with hydra (part of this development effort). This paper will also overview a new mathematical framework [OSS11], [ALL11], [KLL11], [LOO08] for the quantification of uncertainties, which provides a formulation of UQ problems as global optimization problems.

\section{Rigorous Uncertainty Quantification}

Following [LOO08], we specifically take a certification point of view of uncertainty quantification. For definiteness, we consider systems whose operation can be described in terms of $N$ scalar performance measures $\left(Y_{1}, \ldots, Y_{N}\right)=Y \in \mathbb{R}^{N}$. The response of the system is taken as stochastic due to the intristic randomness of the system, or randomness in the input parameters defining the operation of the system, or both. Suppose that the outcome $Y \in A$ constitutes a satisfactory outcome for the system of interest, for some prescribed measureable admissible set $A \subseteq \mathbb{R}^{N}$. Hence, we are interested in determining the probability of failure (PoF) $\mathbb{P}[Y \in$ $\left.A^{c}\right]$.

Evidently, for an upper bound to be useful, it must also be tight (i.e. it must be close to the actual PoF of the system) and accessible by some combination of laboratory and computational means. In [ALL11], [KLL11], a methodology for a rigorous determination of tight upper bounds on the probability of failure for complex systems is presented, and is summarized below.

We consider a response function $Y=F(X)$ that maps controllable system inputs $X$ to performance measures $Y$, and relies on a probability of failure (PoF) upper bounds of the concentration of measure (CoM) type [BBL04], [LED01], [MCD89]. If McDiarmid's inequality [MCD89] (i.e. the bounded differences inequality) is used to bound $\mathrm{PoF}$, the system may then be certified on the sole knowledge of ranges of its input parameters -- without a priori knowledge of their probability distributions, its mean performance $\mathbb{E}[Y]=M$ and a certain measure $D_{G}=U$ of the spread of the response, known as system diameter, which provides a rigorous quantitative measure of the uncertainty in the response of the system.

A model is regarded as $Y=F(X)$ that approximates the response $Y=G(X)$ of the system. An upper bound on the system diameter -- and thus on the uncertainty in the response of the system -- then follows from the triangle inequality $D_{G} \leq D_{F}+D_{G-F}$, and $U=D_{F}+D_{G-F}$ can be taken as a new -- and conservative -- measure of system uncertainty. In this approach, the total uncertainty of the system is the sum of the predicted uncertainty (i.e. the variability in performance predicted by the model as quantified by the model diameter $D_{F}$ ), and the modeling-error uncertainty (i.e. the discrepancy between model prediction and experiment as quantified by the modeling-error diameter $D_{G-F}$.

In [LOO08], PoF upper bounds of the CoM type were formulated by recourse to McDiarmid's inequality. In its simplest version, this inequality pertains to a system characterized by $N$ real random inputs $X=\left(X_{1}, \ldots, X_{N}\right) \in E \subseteq \mathbb{R}^{N}$ and a single real performance measure $Y \in \mathbb{R}$. Suppose that the function $G: \mathbb{R}^{N} \rightarrow \mathbb{R}$ describes the response function of the system. Suppose that the system fails when $Y \leq a$, where $a$ is a threshold for the safe operation of the system. Then, a direct application of McDiarmid's inequality gives the following upper bound on the PoF of the system:

$$
\mathbb{P}[G \leq a] \leq \exp \left(-2 \frac{M^{2}}{U^{2}}\right)
$$

where

$$
M=(\mathbb{E}[G]-a)_{+}
$$

is the design margin and

$$
U=D_{G}
$$

is the system uncertainty. In (3), $D_{G}$ is the diameter of the response function. From (1) it follows that the system is certified if

$$
\exp \left(-2 \frac{M^{2}}{U^{2}}\right) \leq \varepsilon
$$

where $\varepsilon$ is the PoF tolerance, or, equivalently, if

$$
\mathrm{CF}=\frac{M}{U} \geq \sqrt{\log \sqrt{\frac{1}{\varepsilon}}}
$$

where $\mathrm{CF}$ is the confidence factor. In writing (2) and subsequently, we use the function $x_{+}:=\max (0, x)$. We see from the preceding expressions that McDiarmid's inequality supplies rigorous quantitative definitions of design margin and system uncertainty. In particular, the latter is measured by system diameter $D_{G}$, which measures the largest deviation in performance resulting from arbitrarily large perturbations of one input parameter at a time. Within this simple framework, rigorous certification is achieved by the determination of two--and only two--quantities: the mean performance $\mathbb{E}[G]$ and the system diameter $D_{G}$.

McDiarmid's inequality is a result in probability theory that provides an upper bound on the probability that the value of a function depending on multiple independent random variables deviates from its expected value. A central device in McDiarmid's inequality is the diameter of a function. We begin by recalling that the oscillation $\operatorname{osc}(f, E)$ of a real function $f: E \rightarrow \mathbb{R}$ over a set $E \in R$ is

$$
\operatorname{osc}(f, E)=\sup \{|f(y)-f(x)|: x, y \in E\}
$$

Thus, osc $(f, E)$ measures the spread of values of $f$ that may be obtained by allowing the independent variables to range over its entire domain of definition. For functions $f: E \subset \mathbb{R}^{N} \rightarrow \mathbb{R}$ of several real values, component-wise suboscillations can be defined as

$$
\operatorname{osc}_{i}(f, E)=\sup \left\{|f(y)-f(x)|: x, y \in E, x_{j}=y_{j} \text { for } j \neq i\right\}
$$

Thus $\operatorname{osc}_{i}(f, E)$ measures the maximum oscillation among all onedimensional fibers in the direction of the $i$ th coordinate. The diameter $D(f, E)$ of the function $f: E \rightarrow \mathbb{R}$ is obtained as the root-mean square of its component-wise suboscillations:

$$
D(f, E)=\left(\sum_{i=1}^{n} \operatorname{osc}_{i}^{2}(f, E)\right)^{1 / 2}
$$

and it provides a measure of the spread of the range of the function. Thus (6) also us to regard $\operatorname{osc}_{i}(f, E)$ as a subdiameter of the system corresponding to variable $X_{i}$, where the subdiameter can be 
regarded as a measure of uncertainty contributed by the variable $X_{i}$ to the total uncertainty of the system.

The attractiveness of the McDiarmid CoM approach to UQ relies on the requirement of tractable information on response functions (sub-diameters) and measures (independence and mean response). Above, it is described how to "plug" this information into McDiarmid's concentration inequality to obtain an upper bound on probabilies of deviation. One may wonder if it is possible to obtain an "optimal" concentration inequality, especially when the available information may not necessarily be sub-diameters and mean values. A general mathematical framework for optimally quantifying uncertainties based only on available information has been proposed [OSS11], and will be summarized here. Assume, for instance, that one wants to certify that

$$
\mathbb{P}[G \geq a] \leq \varepsilon
$$

based on the information that $\operatorname{osc}_{i}(G, E) \leq D_{i}, X=\left(X_{1}, \ldots, X_{N}\right)$, $\mathbb{E}[G] \leq 0$ and that the inputs $X_{i}$ are independent under $\mathbb{P}$. In this situation, the optimal upper bound $\mathscr{U}\left(\mathscr{A}_{M D}\right)$ on the PoF $\mathbb{P}[G \geq a]$ is the solution of the following optimization problem

$$
\mathscr{U}\left(\mathscr{A}_{M D}\right)=\sup _{(f, \mu) \in \mathscr{A}_{M D}} \mu[f(X) \geq a]
$$

subject to constraints provied by the information set

$$
\mathscr{A}_{M D}=\left\{(f, \mu) \mid \begin{array}{c}
f: E_{1} \times \cdots \times E_{N} \rightarrow \mathbb{R}, \\
\mu \in \mathscr{M}\left(E_{1}\right) \otimes \cdots \otimes \mathscr{M}\left(E_{N}\right), \\
\mathbb{E}_{\mu}[f] \leq 0, \\
\operatorname{osc}_{i}(f, E) \leq D_{i}
\end{array}\right\}
$$

where $\mathscr{M}\left(E_{k}\right)$ denotes the set of measures of probability on $E_{k}$. Hence, McDiarmid's inequality is the statement that

$$
\mathscr{U}\left(\mathscr{A}_{M D}\right) \leq \exp \left(-2 \frac{a^{2}}{\sum_{i=1}^{N} D_{i}^{2}}\right)
$$

Similarly, for any other set of information $\mathscr{A}$, we have an optimal (i.e.) least upper bound on the probability of deviation

$$
\mathscr{U}(\mathscr{A})=\sup _{(f, \mu) \in \mathscr{A}} \mu[f(X) \geq a]
$$

The idea is that in practical applications, the available information does not determine $(G, \mathbb{P})$ uniquely, but does determine a set $\mathscr{A}$ such that $(G, \mathbb{P}) \in \mathscr{A}$ and such that any $(f, \mu) \in \mathscr{A}$ could a priori be $(G, \mathbb{P})$. This mathematical framework, called optimal uncertainty quantification (OUQ), is based on the observation that, given a set of assumptions and information about the problem, there exist optimal bounds on uncertainties; these are obtained as extreme values of well-defined optimization problems corresponding to extremizing probabilities of failure, or of deviations, over the feasible set $\mathscr{A}$. Observe that this framework does not implicitly impose inappropriate assumptions, nor does it repudiate relevant information. Indeed, as demonstrated in (10 and 11) for the CoM approach, OUQ can pose a problem that incorporates the assumptions utilized in other common UQ methods (such as Bayesian inference [LJH99]) and provide a rigorous optimal bound on the uncertainties.

Although some OUQ problems can be solved analytically, most must be solved numerically. To that end, the reduction theorems of [OSS11] reduce the infinite-dimensional feasible set $\mathscr{A}$ to a finite-dimensional subset $\mathscr{A}_{\Delta}$ that has the key property that the objective function $(\mathrm{PoF})$ has the same lower and upper extreme values over $\mathscr{A}_{\Delta}$ as over $\mathscr{A}$.

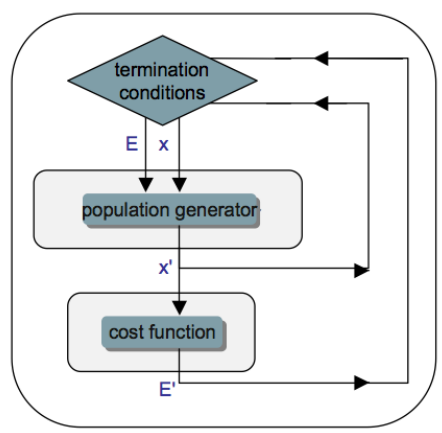

Fig. 1: Conceptual diagram for an optimizer. The cost function provides a difference metric that accepts input parameters $x$ and produces a cost $E$.

For example, the reduction for $\mathscr{A}_{M D}$ in (10) is to pass to measures $\mu=\mu_{1} \otimes \cdots \otimes \mu_{N}$ such that each marginal measure $\mu_{i}$ is supported on at most two points of the parameter space $E_{i}$, i.e. $\mu_{i}$ is a convex combination of two Dirac measures (point masses). Having reduced the set of feasible measures $\mu$, the set of feasible response functions $f$ is also reduced, since we only care about the values of $f$ on the finite support of $\mu$ and nowhere else.

We refer the reader to [OSS11] for the more general reduction theorems. The essential point is that if the information/constraints take the form of $n_{i}$ inequalities of the form $\mathbb{E}_{\mu_{i}}\left[\phi_{j}\right] \leq 0$ (for some test functions $\phi_{j}$ ) and $n^{\prime}$ inequalities of the form $\mathbb{E}_{\mu}\left[\phi_{j}\right] \leq 0$, then it is enough to consider $\mu_{i}$ with support on $1+n_{i}+n^{\prime}$ points of $E_{i}$.

The reduction theorems leave us with a finite-dimensional optimization problem in which the optimization variables are suitable parametrizations of the reduced feasible scenarios $(f, \mu)$.

\section{A Highly-Configurable Optimization Framework}

We have built a robust optimization framework (mystic) [MHA09] that incorporates the mathematical framework described in [OSS11], and have provided an interface to prediction, certification, and validation as a framework service. The mystic framework provides a collection of optimization algorithms and tools that lowers the barrier to solving complex optimization problems. mystic provides a selection of optimizers, both global and local, including several gradient solvers. A unique and powerful feature of the framework is the ability to apply and configure solver-independent termination conditions --- a capability that greatly increases the flexibility for numerically solving problems with non-standard convergence profiles. All of mystic's solvers conform to a solver API, thus also have common method calls to configure and launch an optimization job. This allows any of mystic's solvers to be easily swapped without the user having to write any new code.

The minimal solver interface:

\# the function to be minimized and the initial values from mystic.models import rosen as my_model

$\mathrm{x} 0=[0.8,1.2,0.7]$

\# configure the solver and obtain the solution

from mystic.solvers import fmin

solution = fmin (my_model, $\mathrm{x} 0$ )

The criteria for when and how an optimization terminates are of paramount importance in traversing a function's potential well. Standard optimization packages provide a single convergence condition for each optimizer. mystic provides a set of fully 
customizable termination conditions, allowing the user to discover how to better navigate the optimizer through difficult terrain. Optimizers can be further configured through several methods for choosing the InitialPoints.

The expanded solver interface:

\# the function to be minimized and initial values from mystic.models import rosen as my_model $x 0=[0.8,1.2,0.7]$

\# get monitor and termination condition objects from mystic.monitors import Monitor, VerboseMonitor stepmon = VerboseMonitor(5)

evalmon = Monitor ()

from mystic.termination import ChangeoverGeneration COG = ChangeOverGeneration()

\# instantiate and configure the solver

from mystic.solvers import NelderMeadSimplexSolver solver $=$ NelderMeadSimplexSolver $(l e n(x 0))$

solver.SetInitialpoints ( $x 0$ )

solver.SetGenerationMonitor (stepmon)

solver.SetEvaluationMonitor(evalmon)

solver.Solve (my_model, COG)

\# obtain the solution

solution = solver.bestSolution

\# obtain diagnostic information

function_evals = solver.evaluations

iterations = solver.generations

cost $=$ solver.bestEnergy

\# modify the solver configuration, and continue

COG $=$ ChangeOverGeneration (tolerance $=1 e-8)$

solver.Solve (my_model, COG)

\# obtain the new solution

solution = solver.bestsolution

mystic provides progress monitors that can be attached to an optimizer to track progress of the fitted parameters and the value of the cost function. Additionally, monitors can be customized to track the function gradient or other progress metrics. Monitors can also be configured to record either function evaluations or optimization iterations (i.e. generations). For example, using VerboseMonitor (5) in the SetGenerationMonitor method will print the bestEnergy to stdout every five generations.

\section{Constraints Toolkit}

mystic provides a method to constrain optimization to be within an $\mathrm{N}$-dimensional box on input space, and also a method to impose user-defined parameter constraint functions on any cost function. Thus, both bounds constraints and parameter constraints can be generically applied to any of mystic's unconstrained optimization algorithms. Traditionally, constrained optimization problems tend to be solved iteratively, where a penalty is applied to candidate solutions that violate the constraints. Decoupling the solving of constraints from the optimization problem can greatly increase the efficiency in solving highly-constrained nonlinear problems -- effectively, the optimization algorithm only selects points that satisfy the constraints. Constraints can be solved numerically or algebraically, where the solving of constraints can itself be cast as an optimization problem. Constraints can also be dynamically applied, thus altering an optimization in progress.

Penalty-based methods indirectly modify the candidate solution by applying a change in energy $\Delta E=k \cdot p(\vec{x})$ to the

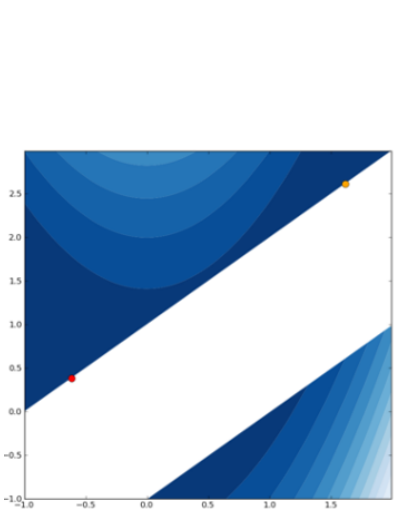

constrained surface contours

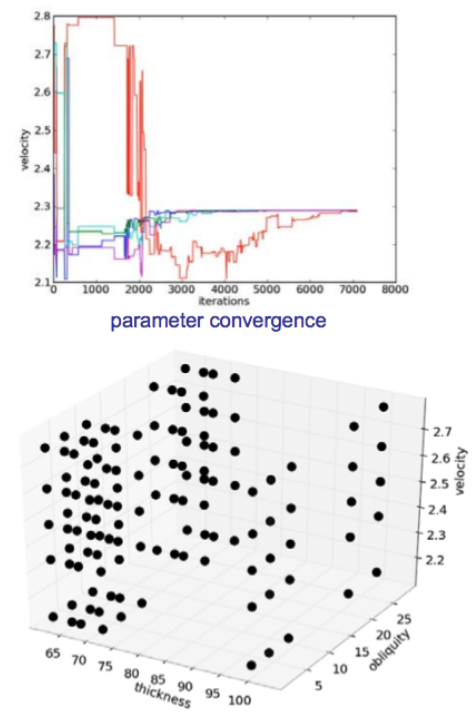

probability distribution over design space

Fig. 2: Optimization analysis viewers available in mystic.

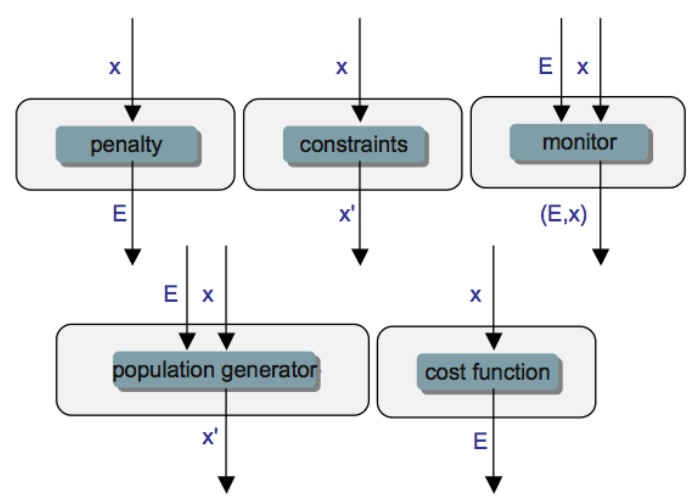

Fig. 3: Basic components provided in the optimizer toolkit. Several wrapper classes are also provided for binding components, while factory classes are provided for generating components.

unconstrained cost function $f(\vec{x})$ when the constraints are violated. The modified cost function $\phi$ is thus written as:

$$
\phi(\vec{x})=f(\vec{x})+k \cdot p(\vec{x})
$$

Set-based methods directly modify the candidate solution by applying a constraints solver $c$ that ensures the optimizer will always select from a set of candidates that satisfy the constraints. The constraints solver has an interface $\vec{x}^{\prime}=c(\vec{x})$, and the cost function becomes:

$$
\phi(\vec{x})=f(c(\vec{x}))
$$

Adding parameter constraints to a solver is as simple as building a constraints function, and using the SetConstraints method. Additionally, simple bounds constraints can also be applied through the SetStrictRanges method:

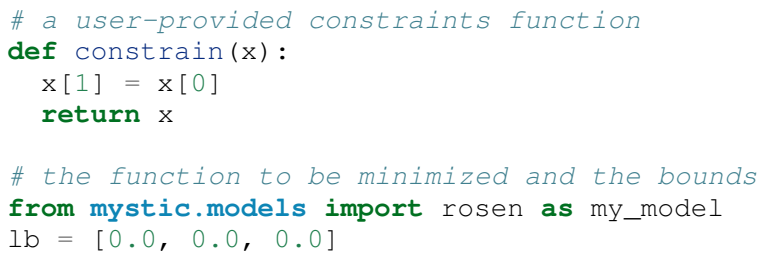




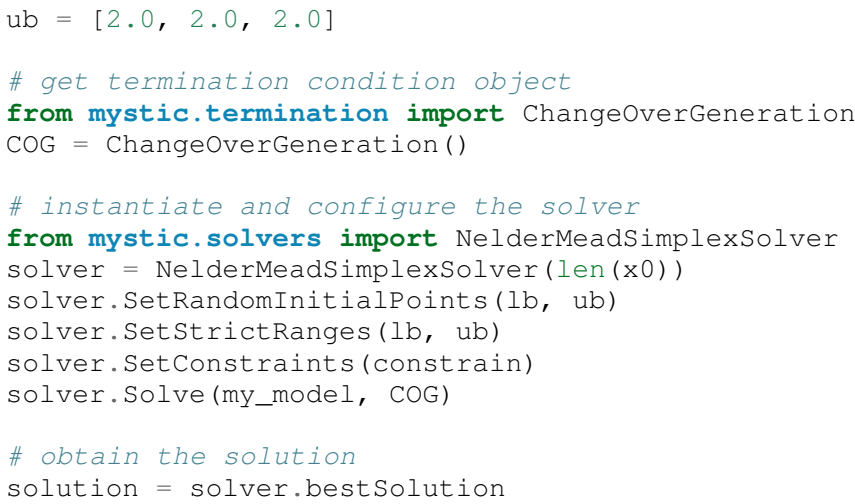

mystic provides a simple interface to a lot of underlying complexity -- thus allowing a non-specialist user to easily access optimizer configurability and high-performance computing without a steep learning curve. This feature must also be applied to the application of constraints on a function or measure. The natural syntax for a constraint is one of symbolic math, hence mystic leverages SymPy [SYM11] to construct a symbolic math parser for the translation of the user's input into functioning constraint code objects:

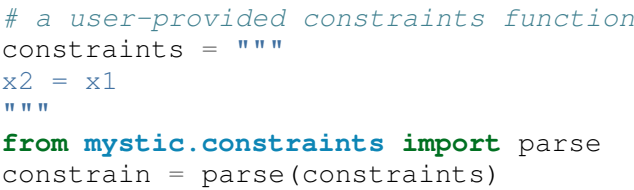

The constraints parser is a constraints factory method that can parse multiple and nonlinear constraints, hard or soft (i.e. " $\sim$ ) constraints, and equality or inequality (i.e. ">") constraints.

Similar tools exist for creating penalty functions, including a SetPenalty method for solvers. Available penalty methods include the exterior penalty function method [VEN09], the augmented Lagrange multiplier method [KSK94], and the logarithmic barrier method [JJB03]. At the low-level, penalty functions are bound to the cost function using mystic's functionWrapper method.

It is worth noting that the use of a constraints solver $c$ does not require the constraints be bound to the cost function. The evaluation of the constraints are decoupled from the evaluation of the cost function -- hence, with mystic, highly-constrained optimization decomposes to the solving of $K$ independent constraints, followed by an unconstrained optimization over only the set of valid points. This method has been shown effective for solving optimization problems where $K \approx 200$ [OSS11].

\section{Seamless Migration to Parallel Computing}

mystic is built from the ground up to utilize parallel and distributed computing. The decomposition of optimization algorithms into their component parts allow this decomposition to not only be in an abstraction layer, but across process-space. mystic provides a modelFactory method that convers a user's model to a service. We define a service to be an entity that is callable by globally unique identifier. Services can also be called by proxy. In mystic, services also include infrastructure for monitoring and handling events. An optimization is then composed as a network of interacting services, with the most common being the user's model or cost function being mapped over parallel resources.

mystic provides several stock models and model factories that are useful for testing:

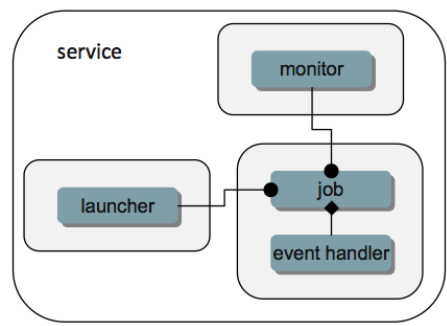

Fig. 4: Conceptual diagram for a service-based model. Here, the job is the fundamental commodity of work, and is the object on which the service is based -- in mystic, this is typically the user's model or a cost function. Services have a global unique identifier, and thus can easily be called by proxy. Note that services may not be located on the machine that requested the service be spawned. Services also can be imbued with infrastructure for monitoring and handling events. Monitors write to a stream that can be piped into another object, such as a logger or one of mystic's viewers.

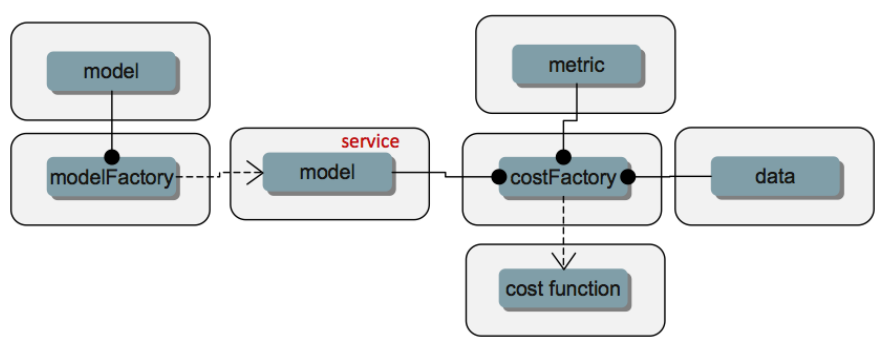

Fig. 5: Use of a modelFactory to cast a user's model $F(x)$ as a service. The model and experimental data $G$ are then bound with a costfactory to produce a cost function. A costFactory can accept a raw user's model, a model proxy, or a model service (as shown here). A typical metric is $|F(x)-G|^{2}$.

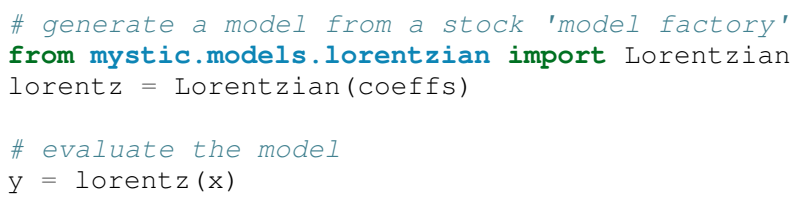

Model factory methods insert pathos infrastructure, thus casting a model as a callable service that has been imbued with pathos infrastructure as shown in Figure (4). The default launcher and map included in mystic are functionally equivalent to execution and map within the standard Python distribution. Any user-provided function can be cast as a service through the use of a modelFactory:

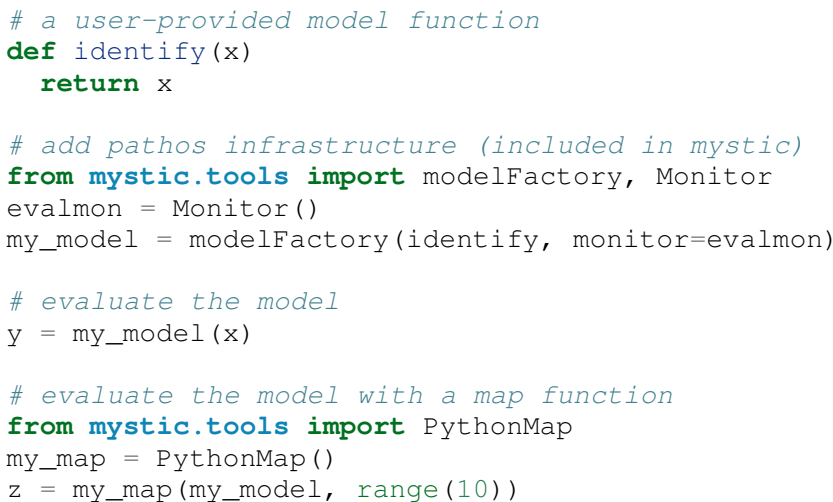




\section{A Framework for Heterogeneous Computing}

We have developed a computational job management framework (pathos) [MBA10] that offers a simple, efficient, and consistent user experience in a variety of heterogeneous environments from multi-core workstations to networks of large-scale computer clusters. pathos provides a single environment for developing and testing algorithms locally -- and enables the user to execute the algorithms on remote clusters, while providing the user with full access to their job history. pathos primarily provides the communication mechanisms for configuring and launching parallel computations across heterogenous resources. pathos provides stagers and launchers for parallel and distributed computing, where each launcher contains the syntactic logic to configure and launch jobs in an execution environment. Some examples of included launchers are: a queue-less MPI-based launcher, a SSHbased launcher, and a multiprocessing launcher. pathos also provides a map-reduce algorithm for each of the available launchers, thus greatly lowering the barrier for users to extend their code to parallel and distributed resources. pathos provides the ability to interact with batch schedulers and queuing systems, thus allowing large computations to be easily launched on highperformance computing resources. One of the most powerful features of pathos is sshTunnel, which enables a user to automatically wrap any distributed service calls within an SSH tunnel.

pathos is divided into four subpackages: dill (a utility for serialization of Python objects), pox (utilities for filesystem exploration and automated builds), pyina (a MPI-based parallel mapper and launcher), and pathos (distributed parallel mapreduce and $\mathrm{SSH}$ communication).

pathos utilizes pyre, which provides tools for connecting components and managing their interactions. The core component used by pathos is a service -- a callable object with a configurable connection mechanism. A service can utilize Launcher and Monitor objects (which provide abstraction to execution and logging, respectively), as well as Strategy objects (which provide abstraction patterns for coupling services). A standard interface for services enables massively parallel applications that utilize distributed resources to be constructed from a few simple building blocks. A Launcher contains the logic required to initiate execution on the current execution environment. The selection of launcher will determine if the code is submitted to a batch queue, run across SSH tunneled RPC connections, or run with MPI on a multiprocessor. A Strategy provides an algorithm to distribute the workload among available resources. Strategies can be static or dynamic. Examples of static strategies include the equalportion strategy and the carddealer strategy. Dynamic strategies are based on the concept of a worker pool, where there are several workload balancing options to choose from. Strategies and launchers can be coupled together to provide higher-level batch and parallel-map algorithms. A Map interface allows batch processing to be decoupled from code execution details on the selected platforms, thus enabling the same application to be utilized for sequential, parallel, and distributed parallel calculations.

\section{Globally Unique Message Passing}

We must design for the case where an optimizer's calculation spans multiple clusters, with a longevity that may exceed the uptime of any single cluster or node. hydra enables any Python object to obtain a network address. After obtaining an address, an object can asynchronously exchange messages with other objects on the network. Through the use of proxy objects, sending messages to remote objects is easy as calling an instance method on a local object. A call to a proxy transparently pickles the function name along with the arguments, packages the message as a datagram, and sends it over the network to the remote object represented by the proxy. On the recieving end, there is a mechanism for responding to the sender of the current message. Since message sending is asynchronous, an object responds to a message by sending another message.

The modelFactory method essentially provides mystic with a high-level interface for a pathos server, with an option to bind a monitor directly to the service. The lower-level construction of a distributed service, using SSH-based communication, is as follows:

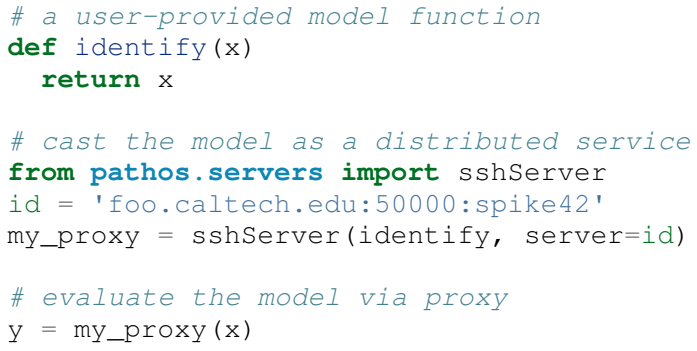

Parallel map functions are built around available launchers, providing a high-level interface to launching several copies of a model in parallel. The creation of a parallel map that will draw from a pool of two local workers and all available IPC servers at 'foo.caltech.edu' is shown below:

\# a user-provided model function

def identify $(x)$

return $\mathrm{x}$

\# select and configure a parallel map

from pathos.maps import ipcPool

my_map $=$ ipcPool $(2$, servers=['foo.caltech.edu'] )

\# evaluate the model in parallel

$z$ = my_map (identify, range(10))

\section{Serialization}

dill extends Python's pickle module for serializing and deserializing Python objects to the majority of the built-in Python and NumPy types. Serialization is the process of converting an object to a byte stream, the inverse of which is converting a byte stream back to a Python object hierarchy.

dill provides the user the same interface as the pickle module, and also includes some additional features. In addition to pickling Python objects, dill provides the ability to save the state of an interpreter session in a single command. Hence, it would be feasible to save a interpreter session, close the interpreter, ship the pickled file to another computer, open a new interpreter, unpickle the session and thus continue from the "saved" state of the original interpreter session.

\section{Filesystem Interaction}

pox provides a collection of utilities for navigating and manipulating filesystems. This module is designed to facilitate some of the low level operating system interactions that are useful when exploring a filesystem on a remote host, where queries such as 


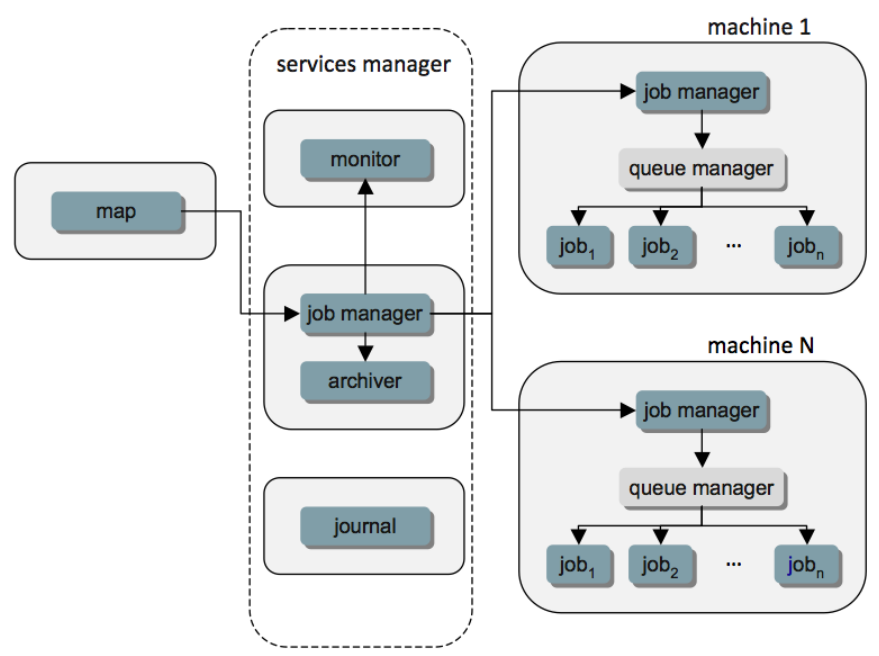

Fig. 6: Conceptual diagram for heterogeneous job management. A distributed parallel map function is used to copy a service $n$ times on $N$ machines. If the object being mapped is not a service, then the services manager is omitted from the diagram -- the jobs still undergo a distributed launch, but are managed at the machine level.

"what is the root of the filesystem?", "what is the user's name?", and "what login shell is preferred?" become essential in allowing a remote user to function as if they were logged in locally. While pox is in the same vein of both the os and shutil built-in modules, the majority of its functionality is unique and compliments these two modules.

pox provides Python equivalents of several unix shell commands such as "which" and "find". These commands allow automated discovery of what has been installed on an operating system, and where the essential tools are located. This capability is useful not only for exploring remote hosts, but also locally as a helper utility for automated build and installation.

Several high-level operations on files and filesystems are also provided. Examples of which are: finding the location of an installed Python package, determining if and where the source code resides on the filesystem, and determining what version the installed package is.

pox also provides utilities to enable the abstraction of commands sent to a remote filesystem. In conjunction with a registry of environment variables and installed utilites, pox enables the user to interact with a remote filesystem as if they were logged in locally.

\section{Distributed Staging and Launching}

pathos provides methods for configuring, launching, monitoring, and controlling a service on a remote host. One of the most basic features of pathos is the ability to configure and launch a IPC-based service on a remote host. pathos seeds the remote host with a small portpicker script, which allows the remote host to inform the localhost of a port that is available for communication.

Beyond the ability to establish a IPC service, and then post requests, is the ability to launch code in parallel. Unlike parallel computing performed at the node level (typically with MPI), pathos enables the user to launch jobs in parallel across heterogeneous distributed resources. pathos provides a distributed map-reduce algorithm, where a mix of local processors and distributed IPC services can be selected. pathos also provides a very basic automated load balancing service, as well as the ability for the user to directly select the resources.

A high-level interface is provided which yields a map-reduce implementation that hides the IPC internals from the user. For example, with ipcPool, the user can launch their code as a distributed parallel service, using standard Python and without writing a line of server or parallel batch code. pathos also provides tools to build a custom Map. In following code, the map is configured to 'autodetect ' the number of processors, and only run on the localhost:

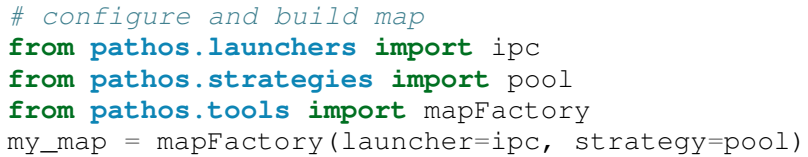

IPC servers and communication in general is known to be insecure. However, instead of attempting to make the IPC communication itself secure, pathos provides the ability to automatically wrap any distributes service or communication in an SSH tunnel. SSH is a universally trusted method. Using sshTunnel, pathos has launched several distributed calculations on clusters at National Laboratories, and to date has performed test calculations that utilize node-to-node communication between two national lab clusters and a user's laptop. pathos allows the user to configure and launch at a very atomistic level, through raw access to s.sh and scp. Any distributed service can be tunneled, therefore less-secure methods of communication can be provided with secure authentication:

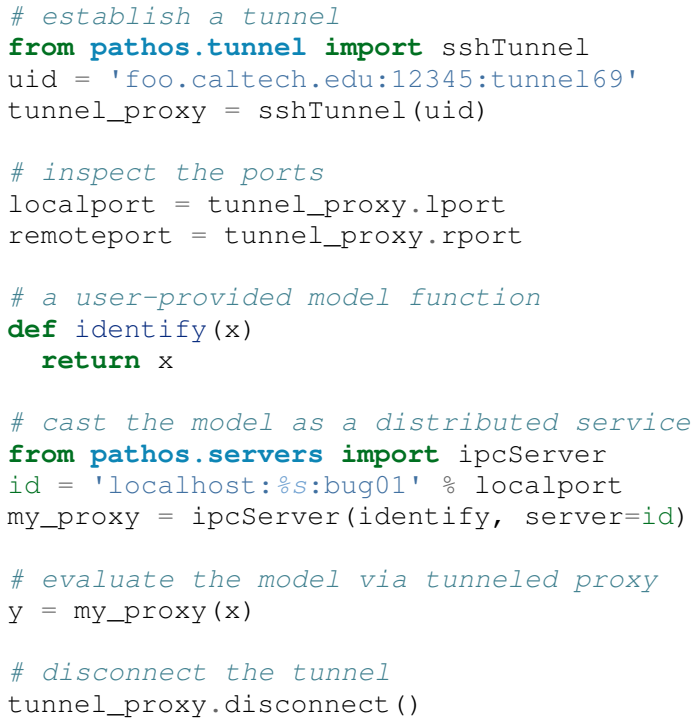

\section{Parallel Staging and Launching}

The pyina package provides several basic tools to make MPIbased high-performance computing more accessable to the end user. The goal of pyina is to allow the user to extend their own code to MPI-based high-performance computing with minimal refactoring.

The central element of pyina is the parallel map-reduce algorithm. pyina currently provides two strategies for executing the parallel-map, where a strategy is the algorithm for distributing the work list of jobs across the availble nodes. These strategies can be used "in-the-raw" (i.e. directly) to provide map-reduce to a user's own MPI-aware code. Further, pyina provides several 
map-reduce implementations that hide the MPI internals from the user. With these Map objects, the user can launch their code in parallel batch mode -- using standard Python and without ever having to write a line of Parallel Python or MPI code.

There are several ways that a user would typically launch their code in parallel -- directly with mpirun or mpiexec, or through the use of a scheduler such as torque or slurm. pyina encapsulates several of these launching mechanisms as Launchers, and provides a common interface to the different methods of launching a MPI job. In the following code, a custom Map is built to execute MPI locally (i.e. not to a scheduler) using the carddealer strategy:

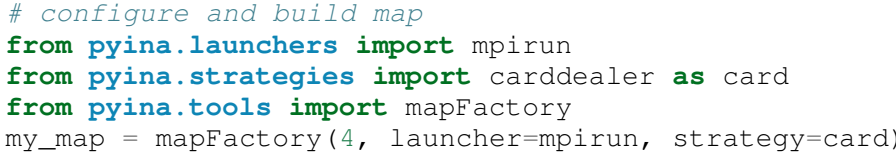

\section{New Massively-Parallel Optimization Algorithms}

In mystic, optimizers have been extended to parallel whenever possible. To have an optimizer execute in parallel, the user only needs to provide the solver with a parallel map. For example, extending the Differential Evolution [SKP95] solver to parallel is involves passing a Map to the SetEvaluationMap method. In the example below, each generation has 20 candidates, and will execute in parallel using MPI with 4 workers:

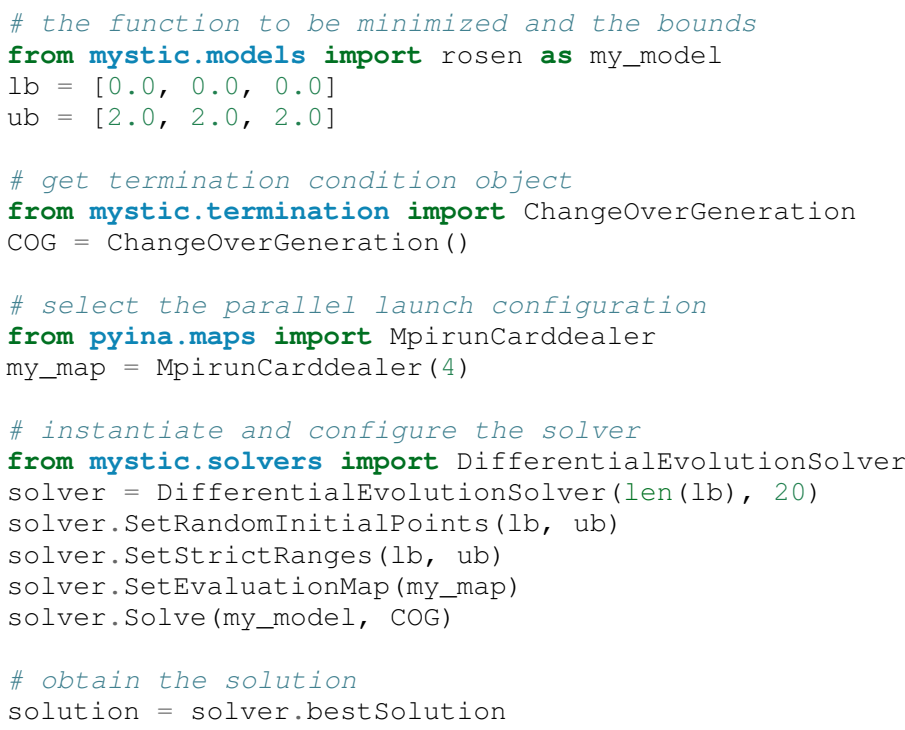

Another type of new parallel solver utilizes the SetNestedSolver method to stage a parallel launch of $N$ optimizers, each with different initial conditions. The following code shows the BuckshotSolver scheduling a launch of $N=20$ optimizers in parallel to the default queue, where 5 nodes each with 4 processors have been requested:

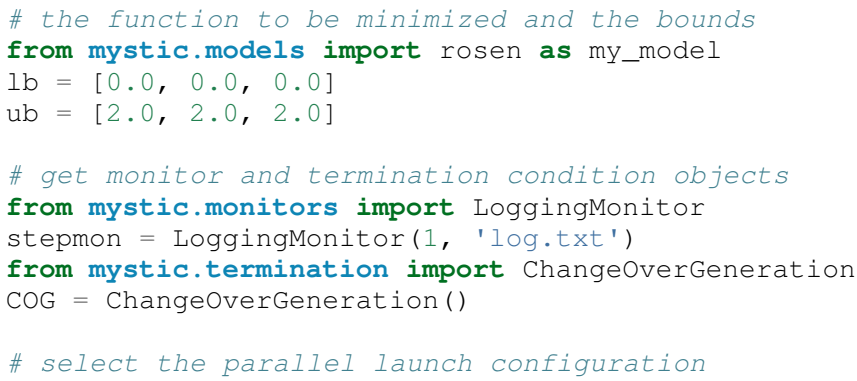

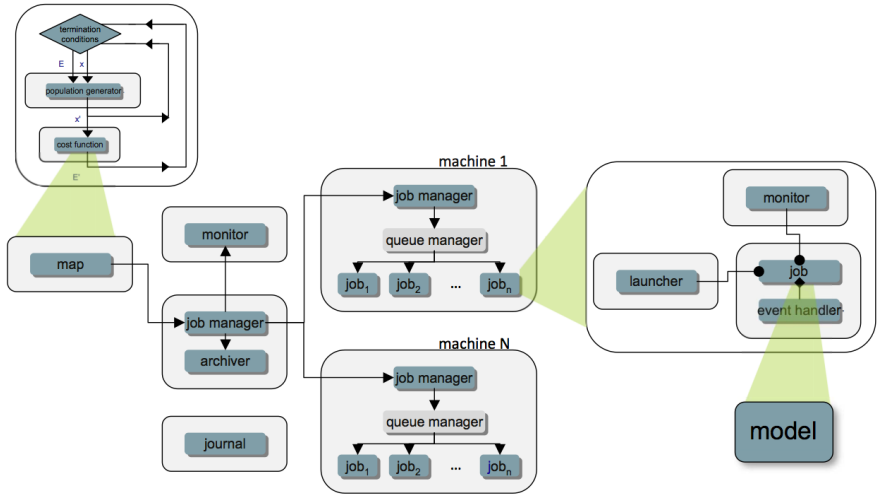

Fig. 7: Conceptual diagram for a carddealer-DE optimizer. The optimizer contains a map function that stages $n$ copies of the user's model $F(x)$ in parallel across distributed resources.

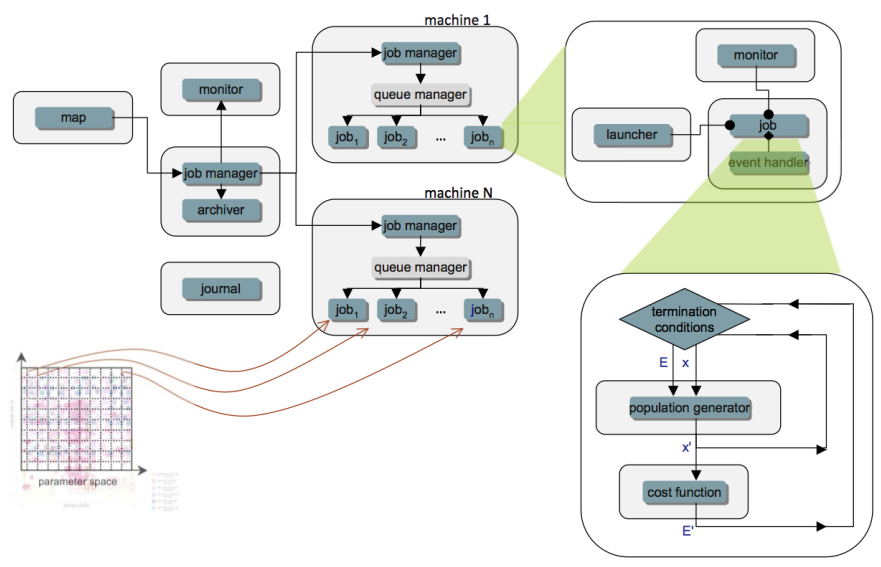

Fig. 8: Conceptual diagram for a lattice-Powell optimizer. $N$ Powell's local-search optimizers are launched in parallel, with each optimizer starting from the center of a different lattice cuboid in parameter space. A buckshot-Powell optimizer is similar; however, instead utilizes a uniform random distribution of initial values.

from pyina.maps import TorqueMpirunCarddealer my_map $=$ TorqueMpirunCarddealer ( $5:$ ppn=4')

\# instantiate and configure the nested solver from mystic.solvers import Powelldirectionalsolver my_solver = PowellDirectionalSolver(len(lb)) my_solver.SetstrictRanges (1b, ub) my_solver.setEvaluationLimits (50)

\# instantiate and configure the outer solver from mystic.solvers import Buckshotsolver solver = BuckshotSolver(len(lb), 20) solver.SetRandomInitialPoints ( lb, ub) solver. SetGenerationMonitor (stepmon) solver. SetNestedSolver(my_solver) solver. SetSolverMap (my_map) solver.solve (my_model, COG)

\# obtain the solution

solution = solver.bestsolution

\section{Probability and Uncertainty Tooklit}

The software framework presented in this paper was designed to solve UQ problems. Calculation of the upper and lower bounds for probability of failure is provided as a framework service. The McDiarmid subdiameter is a model-based measure of sensitivity, 


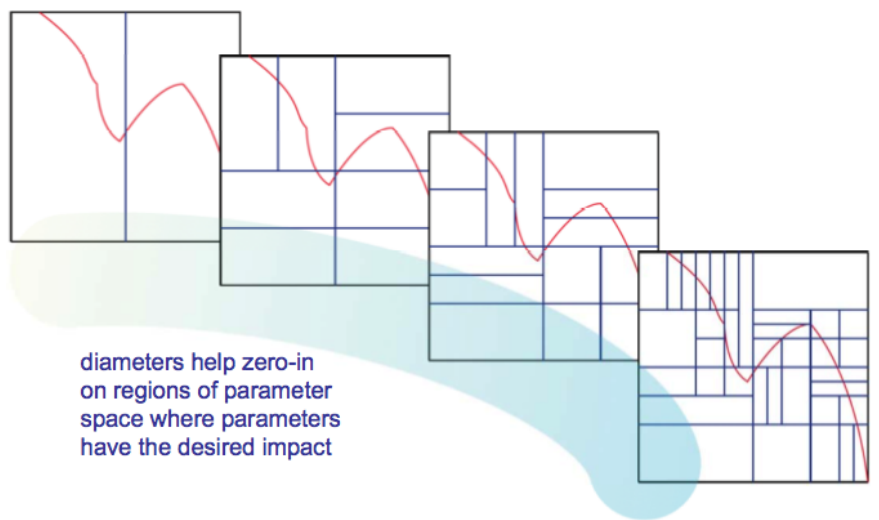

Fig. 9: Coupling an iterative partitioning algorithm with a sensitivity calculation enables the discovery of critical regions in parameter space.

and is cast within mystic as a global optimization. Diameter calculations can be coupled with partitioning algorithms, and used to discover regions of critical behavior. Optimization over probability measures is also available as a framework service, and is utilized in (OUQ) calculations of optimal bounds.

The minimization or maximization of a cost function is the basis for performing most calculations in mystic. The optimizer generates new trial parameters, which are evaluated in a userprovided model function against a user-provided metric. Two simple difference metrics provided are: metric $=|F(x)-G|^{2}$, where $F$ is the model function evaluated at some trial set of fit parameters $\mathscr{P}$, and $G$ is the corresponding experimental data - and metric $=|F(x)-F(y)|^{2}$, where $x$ and $y$ are two slightly different sets of input parameters (6).

mystic provides factory methods to automate the generation of a cost function from a user's model. Conceptually, a costfactory is as follows:

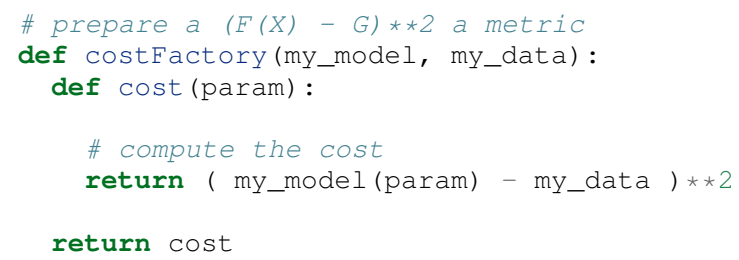

Suboscillations (6), used in calculations of rigorous sensitivity (such as $D_{i} / D$ ), can also be cast as a cost metric:

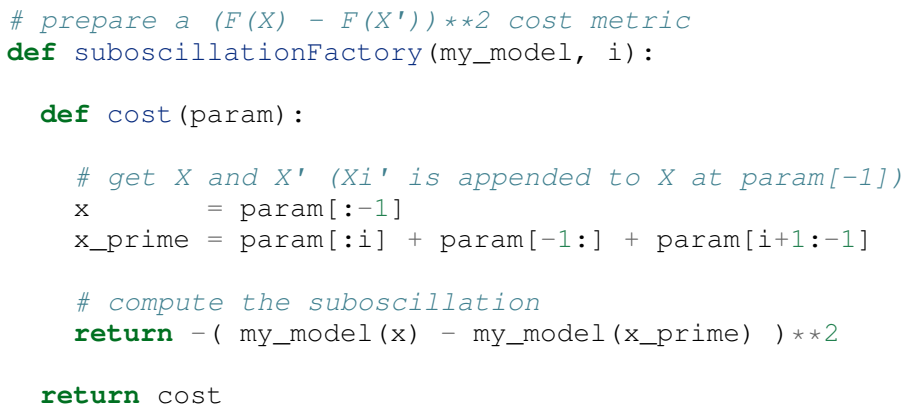

The diameter $D(7)$ is the root-mean square of its componentwise suboscillations. The calculation of the diameter is performed as a nested optimization, as shown above for the BuckshotSolver. Each inner optimization is a calculation of a component suboscillation, using the a global optimizer (such

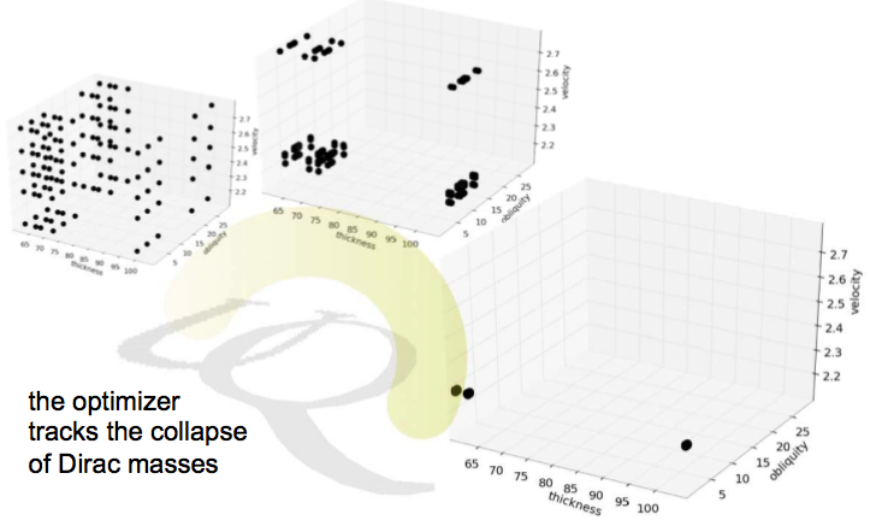

Fig. 10: Optimal uncertainty quantification is an optimization of probability measures over design parameter space. Collapse of probability masses corresponds to the determination of the critical design parameters.

as DifferentialEvolutionSolver) and the cost metric shown above.

The optimization algorithm takes a set of model parameters $\mathscr{P}$ and the current measure of oscillation $O(\mathscr{P})$ as inputs, and produces an updated $\mathscr{P}$. The optimization loop iterates until the termination conditions are satisfied.

When the global optimization terminates the condition $O(\mathscr{P})<-\left(o s c_{i}^{2}+\varepsilon\right)$ is satisfied, and the final set $\mathscr{P}$ is composed of $X$ and $X^{\prime}$.

OUQ problems can be thought of optimization problems where the goal is to find the global maximum of a probability function $\mu[H \leq 0]$, where $H \leq 0$ is a failure criterion for the model response function $H$. Additional conditions in an OUQ problem are provided as constraints on the information set. Typically, a condition such as a mean constraint on $H, m_{1} \leq \mathbb{E}_{\mu}[H] \leq m_{2}$, will be imposed on the maximization. After casting the OUQ problem in terms of optimization and constraints, we can plug these terms into the infrastructure provided by mystic.

Optimal uncertainty quantification (OUQ) is maximization over a probability distribution, and not over a standard difference metric. Therefore the fundamental data structure is not the userprovided model function, but is a user-configured probability measure. For example, a discrete measure is represented by a collection of support points, each with an accompanying weight. Measures come with built-in methods for calculating the mass, range, and mean of the measure, and also for imposing a mass, range, and mean on the measure. Measures also have some very basic operations, including point addition and subtraction, and the formation of product measures.

Global optimizations used in solving OUQ problems are composed in the same manner as shown above for the DifferentialEvolutionsolver. The cost function, however, is not formulated as in the examples above -- OUQ is an optimization over product measures, and thus uses mystic's product_measure class as the target of the optimization. Also as shown above, the bounds constraints are imposed with the SetStrictRanges method, while parameter constraints (composed as below) are imposed with the SetConstraints method. The union set of these constraints defines the set $\mathscr{A}$. 
So for example, let us define the feasable set

$$
\mathscr{A}=\left\{\begin{array}{l|l}
(f, \mu) & \begin{array}{c}
f=\text { my_model }: \prod_{i=1}^{3}\left[\mathrm{lb}_{\mathrm{i}}, \mathrm{ub}_{\mathrm{i}}\right] \rightarrow \mathbb{R}, \\
\mu=\bigotimes_{i=1}^{3} \mu_{i} \in \bigotimes_{i=1}^{3} \mathscr{M}\left(\left[\mathrm{lb}_{\mathrm{i}}, \mathrm{ub}_{\mathrm{i}}\right]\right), \\
\mathrm{m}_{\mathrm{lb}} \leq \mathbb{E}_{\mu}[\mathrm{f}] \leq \mathrm{m}_{\mathrm{ub}}
\end{array}
\end{array}\right\}
$$

which reduces to the finite-dimensional subset

$$
\mathscr{A}_{\Delta}=\left\{\begin{array}{l|l}
(f, \mu) \in \mathscr{A} & \begin{array}{c}
\text { for } \vec{x} \text { and } \vec{y} \in \prod_{i=1}^{3}\left[1 \mathrm{~b}_{\mathrm{i}}, \mathrm{ub}_{\mathrm{i}}\right] \\
\text { and } \vec{w} \in[0,1] \\
\mu_{i}=w_{i} \delta_{x_{i}}+\left(1-w_{i}\right) \delta_{y_{i}}
\end{array}
\end{array}\right\}
$$

where $\vec{x}=$ some $\left(x_{1}, x_{2}, x_{3}\right), \vec{y}=$ some $\left(y_{1}, y_{2}, y_{3}\right)$, and $\vec{w}=$ some $\left(w_{1}, w_{2}, w_{3}\right)$.

To solve this OUQ problem, we first write the code for the bounds, cost function, and constraints -- then we plug this code into a global optimization script, as noted above.

OUQ requires the user provide a list of bounds that follow the formatting convention that mystic's product_measure.load uses to build a product measure from a list of input parameters. This roughly follows the definition of a product measure as shown in equation (16), and also is detailed in the comment block below:

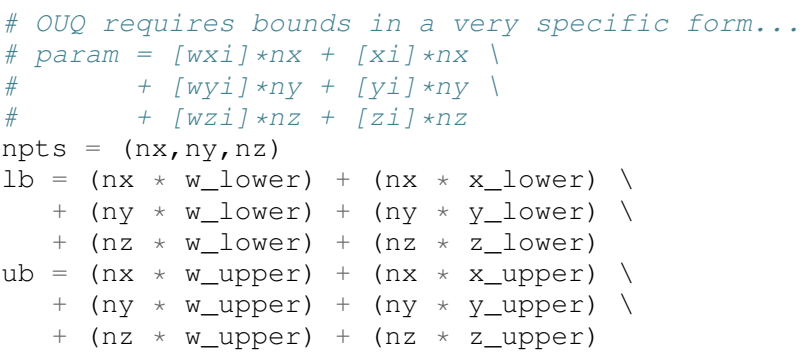

The constraints function and the cost function typically require the use of measure mathematics. In the example below, the constraints check if measure. mass $\approx 1.0$; if not, the the measure's mass is normalized to 1.0. The second block of constraints below check if $m_{1} \leq \mathbb{E}_{\mu}[H] \leq m_{2}$, where $m_{1}=$ target_mean - error and $m_{2}=$ target_mean + error; if not, an optimization is performed to satisfy this mean constraint. The product_measure is built (with load) from the optimization parameters param, and after all the constraints are applied, flatten is used to extract the updated param:

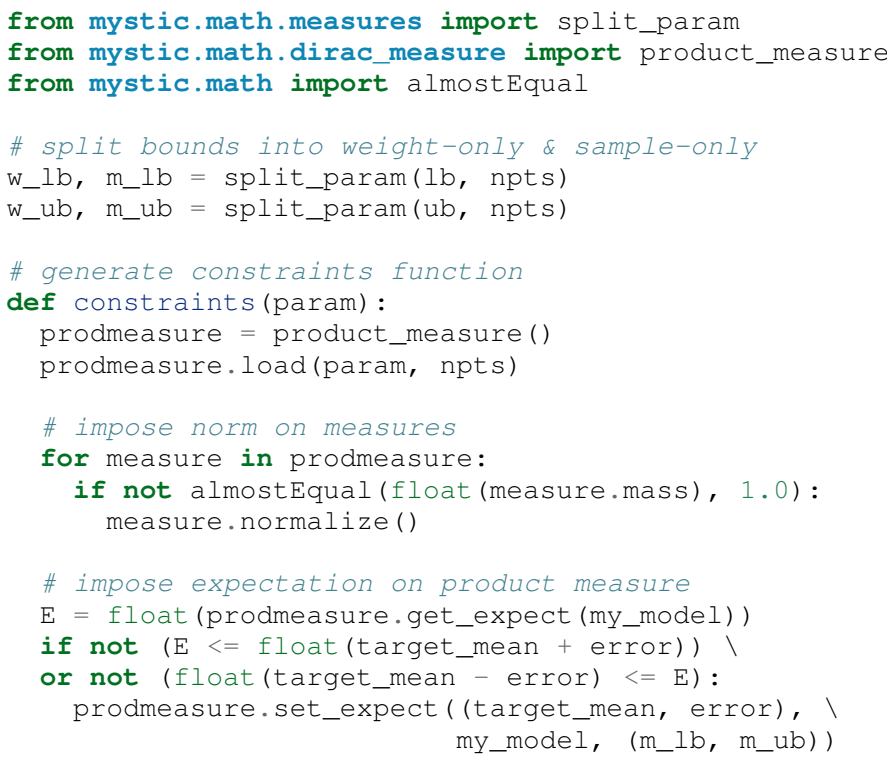

\# extract weights and positions

return prodmeasure.flatten()

The PoF is calculated in the cost function with the pof method:

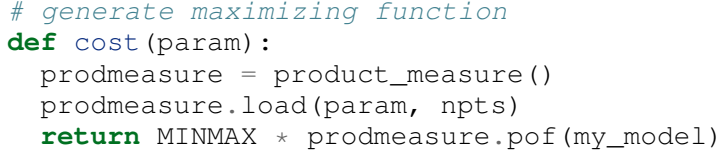

We find the supremum (as in 12) when MINMAX $=-1$ and, upon solution, the function maximum is -solver.bestEnergy. We find the infimum when MINMAX=1 and, upon solution, the function minimum is solver. bestEnergy.

\section{Future Developments}

Many of the features presented above are not currently in released versions of the code. Of primary importance is to migrate these features from development branches to a new release.

The next natural question beyond "what is the sensitivity of a model to an input parameter?" is "how does the correlation between input parameters affect the outcome of the model?". Methods for calculating parameter correlation will be very useful in analysis of results. Another natural question is how to handle uncertainty in the data.

New partitioning algorithms for the discovery of regions of critical behavior will be added to mystic. Currently the only partitioning rule drives the optimizer toward partitioning space such that the upper bounds of a "piecewise-McDiarmid" type are iteratively tightened [STM11]. We will extend the partitioning algorithm not to refine the diameter, but to discover regions where the diameters meet a set of criteria (such as: regions where there are two roughly equal subdiameters that account for $90 \%$ or more of the total diameter (i.e. automated discovery of regions where two parameters compete to govern the system behavior). mystic will also further expand its base of available statistical and measure methods, equation solvers, and also make available several more traditional uncertainty quantification methods. mystic will continue to expand its base of optimizers, with particular emphasis on new optimization algorithms that efficiently utilize parallel computing. mystic currently has a few simple parallel optimization algorithms, such as the Latticesolver and BuckshotSolver solvers; however, algorithms that utilize a variant of game theory to do speculation about future iterations (i.e. break the paradigm of an iteration being a blocker to parallelism), or use parallelism and dynamic constraints to allow optimizers launched in parallel to avoid finding the same minimum twice, are planned. Parallelism in optimization also allows us to not only find the global minima, but to simultaneously find all local minima and transition points -- thus providing a much more efficient means of mapping out a potential energy surface. Solving uncertainty quantification problems requires a lot of computational resources and often must require a minimum of both model evaluations and accompanying experiments, so we also have to keep an eye on developing parallel algorithms for global optimization with overall computational efficiency.

pathos includes utilities for filesystem exploration and automated builds, and a utility for the serialization of Python objects, however these framework services will need to be made more robust as more platforms and more extensive objects and codes are tackled. Effort will continue on expanding the management and platform capabilities for pathos, unifying and hardening 
the map interface and providing load balancing for all types of connections. The high-level interface to analysis circuits will be extended to encompass new types of logic for combining and nesting components (as nested optimizers are utilized in many materials theory codes). Monitoring and logging to files and databases across parallel and distributed resources will be migrated from mystic and added as pathos framework services.

\section{Summary}

A brief overview of the mathematical and software components used in building a software framework for predictive science is presented.

\section{Acknowledgements}

This material is based upon work supported by the Department of Energy National Nuclear Security Administration under Award Number DE-FC52-08NA28613, and by the National Science Foundation under Award Number DMR-0520547.

\section{REFERENCES}

[MHA09] M. McKerns, P. Hung, M. Aivazis, mystic: a simple modelindependent inversion framework, 2009, http://dev.danse. us/trac/mystic.

[MBA10] M. McKerns, M. Aivazis, pathos: a framework for heterogeneous computing, 2010, http://dev.danse.us/trac/pathos.

[LOO08] L. Lucas, H. Owhadi, M. Ortiz, Rigorous verification, validation, uncertainty quantification and certification through concentration-of-measure ineqalities, Computer Methods in Applied Mechanics and Engineering 197, 4591, 2008.

[MCD89] C. McDiarmid, On the method of bounded differences, In: Surveys in combinatorics, 1989, vol. 141 of London Math. Soc. Lecture Note Ser., Cambridge Univ. Press, Cambridge, 148.

[BBL04] S. Boucheron, O. Bousquet, G. Lugosi, Concentration inequalities, In: Advanced Lectures in Machine Learning, 2004, Springer, 208.

[LED01] M. Ledoux, The concentration of measure phenomenon, In: Mathematical Surveys and Monographs, 2001, vol 89, American Mathematical Society.

[ALL11] M. Adams, A. Lashgari, B. Li, M. McKerns, J. Mihaly, M. Ortiz, H. Owhadi, A. Rosakis, M. Stalzer, T. Sullivan, Rigorous model-based uncertainty quantification with application to terminal ballistics, part II: systems with uncontrollable inputs and large scatter, Journal of the Mechanics of Physics and Solids, (submitted).

[KLL11] A. Kidane, A. Lashgari, B. Li, M. McKerns, M. Ortiz, H. Owhadi, G. Ravichandran, M. Stalzer, T. Sullivan, Rigorous model-based uncertainty quantification with application to terminal ballistics, part I: systems with controllable inputs and small scatter, Journal of the Mechanics of Physics and Solids, (submitted).

[OSS11] H. Owhadi, C. Scovel, T. Sullivan, M. McKerns, M. Ortiz, Optimal uncertainty quantification, SIAM Review, (submitted).

[VEN09] P. Venkataraman. Applied Optimization with MATLAB Programming. Hoboken, NJ: John Wiley \& Sons, 2009.

[KSK94] B. Kanna, S. Kramer, An Augmented Lagrange Multiplier Based Method for Mixed Integer Discrete Continuous Optimization and Its Applications to Mechanical Design. J. Mech. Des. June 1994. Volume 116, Issue 2, 405. DOI:10.1115/1.2919393.

[JJB03] P. Jensen, J. Bard, Algorithms for Constrained Optimization. Supplement to: Operations Research Models and Methods, 2003, http://www.me.utexas.edu/ jensen/ ORMM/supplements/units/nlp_methods/const_opt.pdf.

[SYM11] O. Certik, et al, SymPy: Python Library for Symbolic Mathematics, http://code.google.com/p/sympy.

[SKP95] R. Storn and K. Price. Differential Evolution: A Simple and Efficient Adaptive Scheme for Global Optimization Over Continuous Spaces. TR-95-012. 1995.
[DAKUQ] M. Eldred, A. Giunta, B. van Bloemen Waanders, S. Wo-

[KROOO] D. Kroshko, et al, OpenOpt, http://openopt.org/

[JOP01] E. Jones, T. Oliphant, P. Peterson, et al, SciPy: Open Source Scientific Tools for Python, 2001, http://www.scipy.org/

[DAKOT] B. Adams, W. Bohnhoff, K. Dalbey, J. Eddy, M. Eldred, D. Gay, K. Haskell, P. Hough, L. Swiler, DAKOTA, A Multilevel Parallel Object-Oriented Framework for Design Optimization, Parameter Estimation, Uncertainty Quantification, and Sensitivity Analysis: Version 5.0 User's Manual, Sandia Technical Report SAND2010-2183, December 2009. jtkiewicz, W. Hart, M. Alleva, DAKOTA, A Multilevel Parallel Object-Oriented Framework for Design Optimization, Parameter Estimation, Uncertainty Quantification, and Sensitivity Analysis. Version 3.0 Developers Manual, Sandia Technical Report SAND2001-3514, April 2002.

[MAGA1] M. Aivazis, et al, pyre: an integration framework for high performance computing, http://danse.us/trac/pyre.

[GVRPY] G. van Rossum, et al, Python Programming Language, http: //www.python.org/

[MPROC] R. Oudkerk, multiprocessing, http://pyprocessing.berlios.de/ [MPI4P] L. Dalcin, mpi4py: MPI for Python, http://mpi4py. googlecode.com/

[VVPPP] V. Vanovschi, pp: Parallel Python Software, http://www. parallelpython.com/

[NUMPY] T. Oliphant, et al, NumPy, http://www.numpy.org/

[MATPL] J. Hunter, Matplotlib: A 2D Graphics Environment, Journal of Computing in Science and Engineering, vol 9(3), 2007

[EKL02] I. Egorov, G. Kretinin, I. Leshchenko, S. Kuptzov, IOSO Optimization Toolkit - Novel Software to Create Better Design, 9th AIAA/ISSMO Symposium on Multidisciplinary Analysis and Optimization, 04 - 06 Sep. 2002, Atlanta, Georgia. http://www.iosotech.com/publicat.htm

[BMM10] S. Benson, L. Curfman McInnes, J. More, T. Munson, J. Sarich, TAO User Manual (Revision 1.10.1), 2010, Mathematics and Computer Science Division Argonne National Laboratory ANL/MCS-TM-242, http://www.mcs.anl.gov/ tao

[STM11] T. Sullivan, U. Topcu, M. McKerns, H. Owhadi, Uncertainty quantification via codimension-one partitioning, International Journal for Numerical Methods in Engineering, 85, 1499 (2011)

[LJH99] T. Leonard, J. Hsu. Bayesian methods, In: volume 5 of Cambridge Series in Statistical and Probabilistic Mathematics. Cambridge University Press, Cambridge, 1999. An analysis for statisticians and interdisciplinary researchers.

[MAT09] The MathWorks Inc., Technical Report 91710v00, March 2009. 